\title{
Liquid Metal Particle Popping: Macroscale to Nanoscale
}

\author{
Trevor R. Lear ${ }^{\dagger}$, Seok-Hee Hyun ${ }^{\dagger}$, John William Boley+, Edward L. White ${ }^{+}$, David H. Thompson ${ }^{\dagger}$, \\ and Rebecca K. Kramer ${ }^{*}$
}

${ }^{\ddagger}$ School of Mechanical Engineering, Purdue University, 585 Purdue Mall, West Lafayette, IN 47907, USA

'Department of Chemistry, Purdue University, 560 Oval Drive, West Lafayette, IN 47907, USA

\begin{abstract}
Liquid metal nanoparticles can be used to produce stretchable electronic devices. Understanding the mechanical properties of liquid metal nanoparticles is crucial to optimizing their use in various applications, especially printing of flexible, stretchable electronics. Smaller nanoparticles are desired for high-resolution printing and compatibility with existing scalable manufacturing methods; however, they contain less liquid metal and are more difficult to rupture than larger particles, making them less desirable for post-processing functionality. This study investigates the mechanics of liquid metal particle rupture as a function of particle size. We employ compression of particle films to characterize the composition of the particle core and derive a minimum particle size required to achieve sintering and subsequent conductance. We further derive the force required to rupture a single particle and validate the results by rupturing individual nanoparticles using atomic force microscopy. Finally, we relate the liquid metal nanoparticles to isotropically-elastic thin-shell microspheres to approximate the particle shell stiffness. An increased understanding of the behavior of liquid metal nanoparticles during rupture reveals limitations of current manufacturing processes and paves the way for the next generation of scalable mass-producible soft electronics using additive manufacturing technologies.
\end{abstract}

Keywords: Gallium indium alloy, liquid metal, nanoparticles, atomic force microscopy, particle rupture, nanoindentation

\section{INTRODUCTION}

Liquid metals offer new opportunities for flexible, stretchable, and shape changing electrical components ${ }^{[1-10]}$. Effective techniques to process liquid metal have been demonstrated and include injection into microchannels ${ }^{[1,3]}$, imprinting ${ }^{[11]}$, masked deposition ${ }^{[12]}$, and extrusion ${ }^{[13-}$ ${ }^{15]}$. Although it is possible to manipulate liquid metals at submillimeter length scales, these techniques are greatly inhibited by the spontaneous formation of a thin metal oxide layer on the liquid metal surface in the presence of oxygen. This metal oxide is the mechanism behind the unique capability of liquid metals to form free-standing structures ${ }^{[14,16]}$, but also produces a high surface tension that makes them incompatible with scalable liquid processing techniques ${ }^{[16,17]}$, such as inkjet printing. Inkjet printing is desirable due to its capacity to create high resolution patterned devices while remaining a high-yield process. The ability to inkjet print any liquid for a particular application is indicated by the Ohnesorge number, which relates the viscous forces to inertial and surface tension forces, and liquid metal has been shown to be outside the range of printability ${ }^{[18]}$. 
We have recently demonstrated the ability to inkjet-print liquid metals by dispersing liquid metal nanoparticles in a printable carrier solvent, which allows us to leverage the carrier solvent properties during processing and the liquid metal properties post-deposition ${ }^{[19]}$. The liquid metal nanoparticles are electrically insulated by an oxide shell that may be ruptured via application of pressure to sinter the particles and form an electrical path. However, optimal particle preparation conditions for compatibility with drop-on-demand printing and functionality post-processing are contrasting: particle size should be minimized for compatibility with printing to avoid nozzle clogging, while particle size should be maximized for electrical functionality to increase the ratio between conductive liquid metal and semi-conductive metal oxide after particle sintering.

Here, a detailed characterization of liquid metal particle sintering through particle rupture is described. We characterize the electrical response of liquid metal nanoparticle films using compression testing and derive a minimum particle size required to achieve sintering and subsequent sheet conductance. We further derive the force required to rupture a single particle as a function of particle size and introduce a composition model for the liquid metal particle core and corresponding metal oxide shell thickness. These results are validated using an atomic force microscopy (AFM) nanoindentation method, through which the individual particles are ruptured with an AFM tip. This work suggests that at sub-micron scales the repeated formation of the metal oxide shell around the liquid metal particles may greatly alter the composition of the particle core. The inherent relationships between particle formation, core and shell composition, and conductance after coalescence are expected to enable scalable manufacturing of liquid metalbased soft electronics. 


\section{NANOPARTICLE FORMATION AND CHARACTERIZATION}

In an approach similar to that of Hohman et al. ${ }^{[17]}$ and revised by Boley et al. ${ }^{[19]}$, we created stable ethanolic nanoscale liquid metal colloids using sonication, which induces mechanical separation to form liquid metal nanoparticles due to the presence of high oscillating shear forces, as shown in Figure 1a. The liquid metal employed in this work is a commercially available eutectic gallium-indium (EGaIn; $78 \% \mathrm{Ga}$ and $21.4 \%$ In by mass; $15.5{ }^{\circ} \mathrm{C}$ melting point, Figure $\mathrm{S} 1)$. Due to exposure to oxygen during the sonication process, gallium oxide continuously forms, fractures, and reforms, which results in decreasing particle size with increasing sonication time (Figure 1b). Similar to the gallium oxide that forms on pure gallium particles ${ }^{[20]}$, the semi-solid oxide layer is electrically insulating and mechanically stabilizing, which prevents spontaneous particle coalescence ${ }^{[21-24]}$. After sonication, a broad distribution of particle geometries were observed ${ }^{[25,26]}$. Previous experiments have employed thiols (for example, 3-mercapto-Nnonylpropionamide (1ATC9)) and polyvinylpyrrolidone (PVP) to achieve geometric and size uniformity, as well as encourage proper suspension ${ }^{[17,19]}$. Here, we examine liquid metal nanoparticles with oxide coatings and thiol coatings to characterize the mechanical and electrical properties of both types of particles.

Using identical methods to Boley et al. ${ }^{[19]}$, we produced liquid metal nanoparticles both with and without thiols with sonication times ranging from 30 minutes to 960 minutes. The particle sizes were characterized using scanning electron microscopy (SEM), with selected samples shown in Figure 1c (SEM images for all samples used can be found in the Supporting Information). Previous results indicated the average particle size started to plateau at slightly above $200 \mathrm{~nm}$ for particles without stabilization agents (thiols); however, those studies did not explore sonication times beyond $120 \mathrm{~min}{ }^{[19]}$. Samples generated by significantly longer sonication times returned a much smaller asymptotic average particle size of approximately $50 \mathrm{~nm}$, as Figure $1 \mathrm{~b}$ shows for thiolated and non-thiolated particles. As seen in Figure 1c, samples sonicated for shorter periods of time (e.g., $30 \mathrm{~min}, 90 \mathrm{~min}$ ) display a larger variance in particle size, with average diameters decreasing with increasing sonication time. It can also be seen that the presence of thiols in solution aids in the formation of smaller, more monodisperse particles; the particle size distribution in non-thiolated thiolated samples shown in Figure 1d is much greater than that of thiolated samples shown in Figure 1e. 


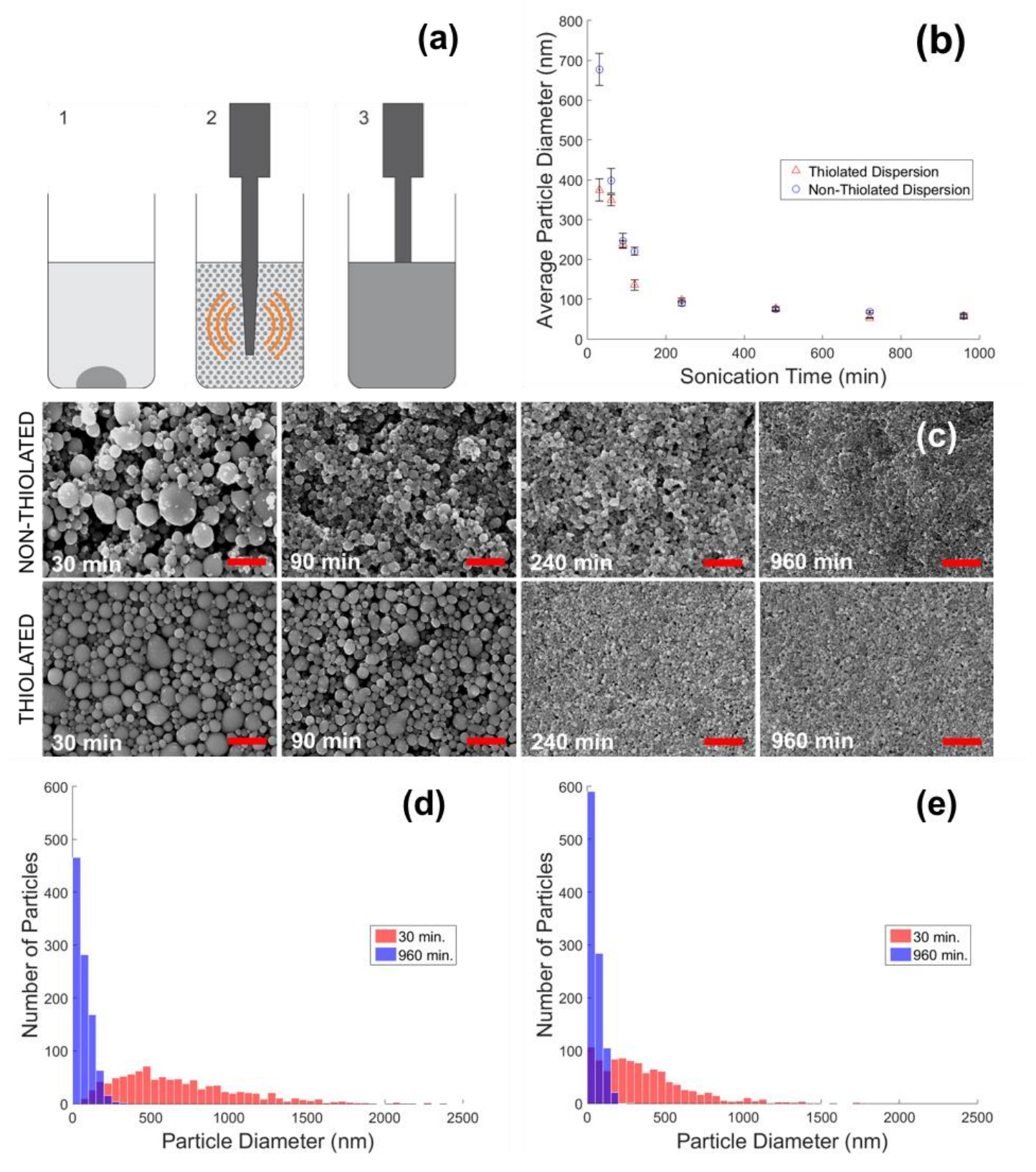

Figure 1. (a) Schematic showing the nanoparticle fabrication process. (b) Average particle diameters for thiolated and non-thiolated dispersions as a function of sonication time. Error bars shown represent one standard deviation. (c) SEM images of non-thiolated and thiolated particles at various sonication times. Scale bar represents $2 \mu \mathrm{m}$. (d)(e) Histograms showing a large polydisperse distribution of particle sizes for the 30 min sonication samples and significantly narrower size distribution for samples sonicated for $960 \mathrm{~min}(\mathrm{n}=1000)$ for non-thiolated and thiolated particles, respectively. 


\section{PARTICLE RUPTURE EXPERIMENTS \& RESULTS}

\section{Particle Film Rupture}

Particle film compression tests were used to determine the relationship between mean particle size and the pressure required to sinter the particles. Mechanical sintering of a film of liquid metal particles has been previously studied ${ }^{[19]}$, and here we extend this work to include both thiolated and non-thiolated particles over a greater range of sizes. Using a single column materials tester, thin films of liquid metal nanoparticles were compressed using two parallel plates while simultaneously measuring the sheet resistance, as shown in Figure 2a. The rupture forces of the particles found using these experiments are represented in Figure $2 b$ which compares the approximate rupture force per particle versus the average particle diameter. Figures $2 \mathrm{c}$-d show raw data for both the non-thiolated and thiolated particles. The curves consistently exhibit the following pattern as the particle films are compressed; (1) a non-conductive state as the flat plate contacts and begins compression of the film, (2) a jump in voltage that signifies successful sintering of the particle film, (3) a continued conductive phase as the film is further compressed, and (4) a saturation state at which compression forces ink out from underneath the plates and breaks the conductive path. Some noise is visible in the data, particularly during the conductive phase; we expect this is due to a combination of polydispersion of the particles and the complex load distributions between particles, both of which may lead to some particles in the film rupturing before others.

As seen in Figures 2c-d, although the thiolated and non-thiolated particles were sonicated for identical durations, the thiolated particles were smaller and more monodisperse, and therefore required greater forces to achieve conductance. We also note that data for films comprised of smaller particles $(\mathrm{d}<70 \mathrm{~nm})$ are not available, as those particle films did not become conductive over the range of forces applied. Using data from these experiments, a relationship between particle size and the required force for sintering was developed (Figure 2b). As detailed in previous work ${ }^{[19]}$, the approximate rupture force per particle, $f$, can be calculated as:

$$
f=\frac{\mathrm{Fd}^{2}}{\mathrm{pD}^{2}}
$$

where $\mathrm{F}$ is the applied force required to sinter the particle film, $\mathrm{D}$ is the diameter of the flat polymethyl methacrylate punch used to compress the particles $(4.35 \pm 0.3 \mathrm{~mm}), \mathrm{d}$ is the average particle diameter, and $\mathrm{p}$ is the areal packing factor for the particles (assumed to be a constant $0.82^{[27]}$. The resulting rupture force per particle as a function of particle diameter shows linear dependence, which agrees with similar studies focused on rupture of the rigid shell around fluidfilled microcapsules ${ }^{[28,29]}$. The smaller variance in particle size for smaller mean particle diameters is reflected in Figure $2 b$, as the error bars decrease with decreasing particle size. Figure $2 \mathrm{~b}$ also shows that the particle rupture force for thiolated and non-thiolated particles is similar for small particles and diverges with increasing particle size. This implies that the mechanical properties of the thiol coating and the metal-oxide coating converge at small particle sizes $(<100 \mathrm{~nm})$. 


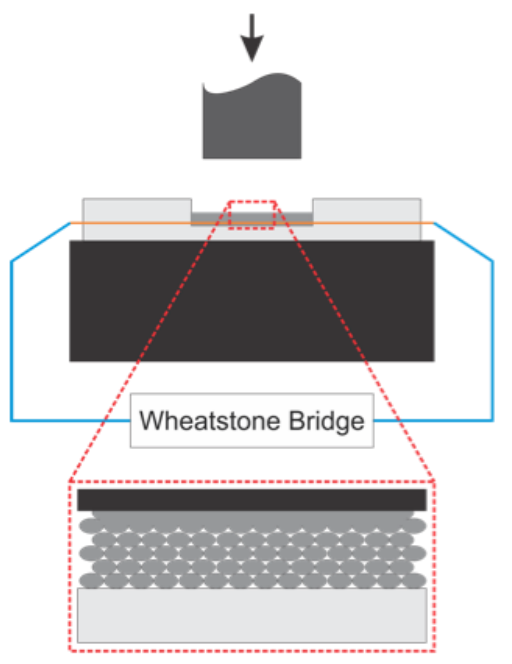

(a)
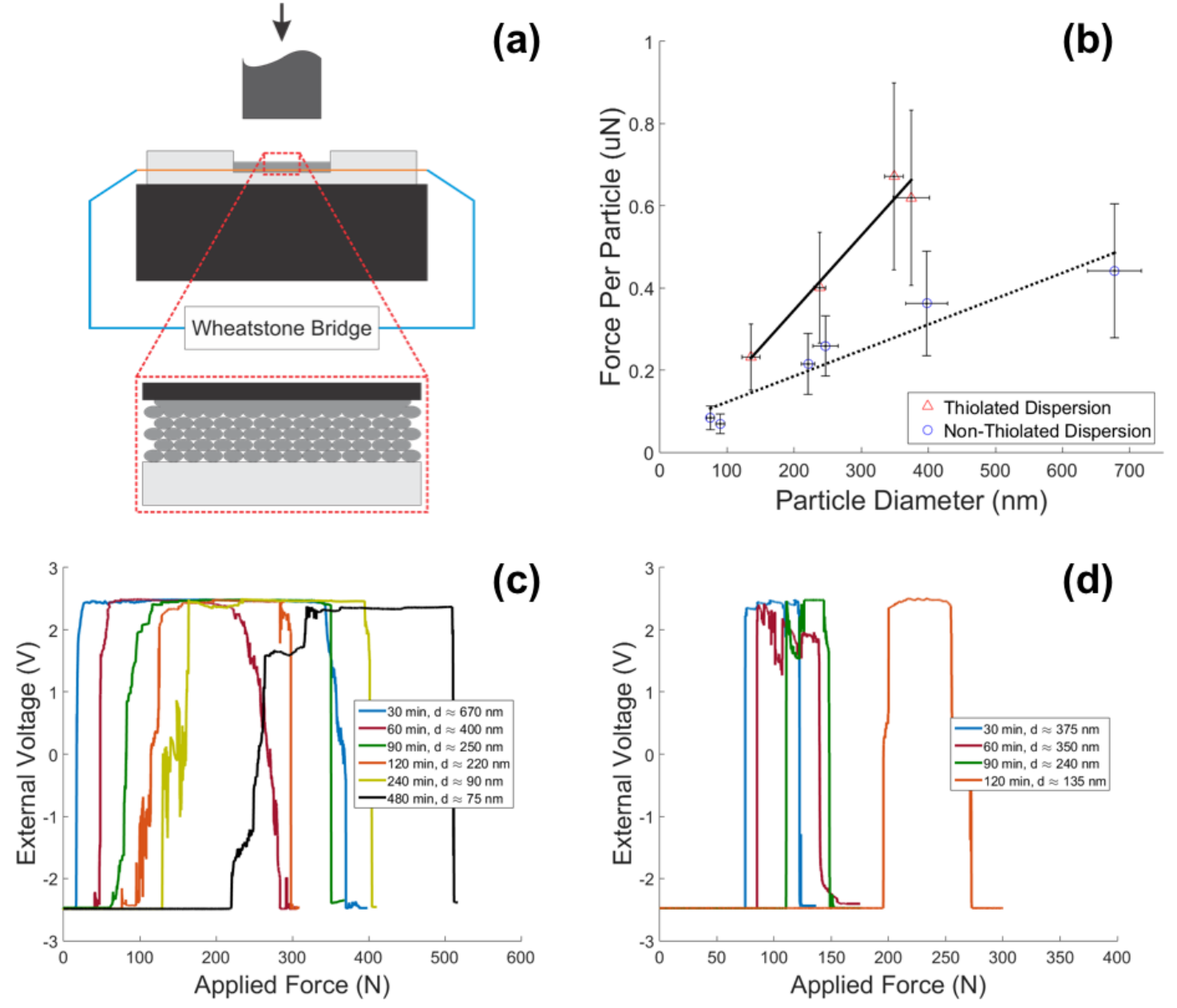

Figure 2. (a) Compression testing of liquid metal nanoparticles using a materials tester. Liquid metal nanoparticle dispersion $(50 \mu \mathrm{L})$ is deposited into a PDMS reservoir fitted with copper wires to measure the resistance during compression. The coalescence of particles is represented in the inset, where particle packing orientation and density are not always known. (b) Particle film compression data - average rupture force per particle vs. mean particle diameter. Error bars represent one standard deviation $(n=5)$. Linear approximation shown. (c) (d) Compression experiment data for non-thiolated and thiolated particles, respectively. 


\section{Particle Core Composition}

Let us consider a single gallium-indium liquid metal nanoparticle under sonication. We assume that the initial composition of the nanoparticle is $78.6 \% \mathrm{Ga}$ and $21.4 \%$ In by mass (determined by the eutectic point of gallium-indium alloy, phase diagram shown in Figure S1), that the mass of the metal is conserved, and that gallium oxide formation on the surface of the nanoparticle contains no trace indium. As gallium oxide forms on the surface of the nanoparticle, gallium composition in the particle core decreases and the core becomes indium-enriched. Figure 3a shows the relationship between particle size, gallium oxide thickness, and the percent of gallium in the particle core. We note that the gallium indium phase diagram shows that a gallium indium alloy will no longer be liquid at room temperature for gallium compositions less than $73 \%$. Therefore, our model indicates a threshold at which the particle core composition will transition from liquid to solid at room temperature. As noted previously, we were unable to experimentally sinter particles with diameters less than $70 \mathrm{~nm}$. Inspecting our model at this minimum rupture threshold, we see that the liquid-solid core transition for $70 \mathrm{~nm}$ diameter particles corresponds to an oxide thickness of approximately $3 \mathrm{~nm}$. Multiple studies have attempted to quantify the thickness of the gallium oxide shell using X-ray scattering and/or photoelectron spectroscopy, resulting in a range from $\sim 0.5 \mathrm{~nm}$ to $5 \mathrm{~nm}{ }^{[20,30,31]}$. Lin et al. and Ren et al. recently performed studies using TEM and quantified the oxide thickness of liquid metal nanoparticles at $3 \mathrm{~nm}$ for

similarly sized particles ${ }^{[32,33]}$, which supports the prediction of our model. Our model further predicts that liquid metal particles with diameters less than $70 \mathrm{~nm}$ and an oxide thickness of $3 \mathrm{~nm}$ should have solid cores, which aligns with our previous observation that particle films comprised of small particles $(\mathrm{d}<70 \mathrm{~nm})$ cannot be sintered at room temperature.

We validated our model via compression experiments using particles that were approximately 55 $\mathrm{nm}$ in diameter, which is less than the predicted $70 \mathrm{~nm}$ threshold that the model suggests will produce solid-core particles under these fabrication conditions. We compressed (sintered) particle films in a materials tester at ambient temperature, where the particle core is predicted to be solid, and an elevated temperature above the melting point of the solid gallium indium alloy core. For the elevated temperature tests, the particle films were deposited onto a plate heated to $50{ }^{\circ} \mathrm{C}$ and allowed to sit for approximately 10 minutes to allow the temperature of the films to stabilize. The films were then compressed using the same parameters as the original film compression tests. As visible in Figure 3b, heated particle films were successfully sintered by applying between $\sim 300$ to $350 \mathrm{~N}$ of force, whereas particle films at ambient temperatures continued to record no electrical response. This result is consistent with our hypothesis that long sonication times result in small and solid-core particles that cannot be ruptured in ambient conditions. 

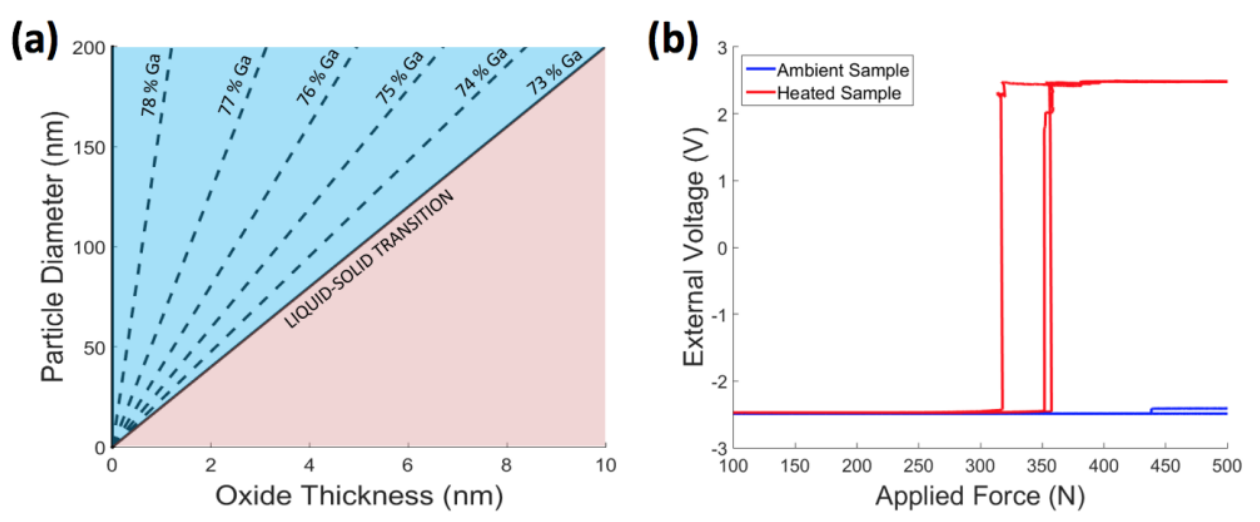

Figure 3. (a) Model relating particle size, gallium oxide thickness, and percent gallium composition in the particle core. Blue region represents particles with liquid cores (which may be ruptured), pink region represents particles with solid cores (which cannot be ruptured). Equation of approximate phase transition line is $D=20.0072 t$ where $D$ is the particle diameter $(\mathrm{nm})$ and $\mathrm{t}$ is the oxide shell thickness $(\mathrm{nm})$. (b) Results of film compression testing of $\sim 55$ $\mathrm{nm}$ particles during heating, activation only achieved for samples heated during compression $(\mathrm{n}=3)$.

\section{Single Particle Rupture}

In order to validate the rupture force per particle as derived from the bulk particle film compression data, we sought to conduct rupture experiments on individual nanoparticles using atomic force microscopy (AFM). To isolate single particles, we first created a monolayer of nanoparticles using the Langmuir-Schaefer method, as seen in Figure 4a (process detailed in Figure S4) [34-37]. Similar experiments have shown that AFM compression of microcapsule monolayers does not differ from compression of isolated microcapsules ${ }^{[38]}$. Because uniform particle size and geometry are desired to produce a monolayer, thiolated particles were used to encourage monodispersion ${ }^{[17]}$. All particles were sonicated for the same duration and underwent differential centrifugation to eliminate extreme variation in particle size, aiding in the formation of a relatively uniform particle monolayer. Figures 4b-c show AFM height and phase maps that confirm the popping of an individual liquid metal nanoparticle, which was further verified using SEM, a technique that has previously been used to identify the rupture of filled particles ${ }^{[39,40]}$.

A single particle rupture experiment is shown in Figures 4d-e and comprises of the following sequence: (1) The AFM tip ( $5 \mathrm{~nm}$ at the thickest point) is lowered until contact is made with the particle shell. (2) Variation in force can be seen in the early regions of contact before the particle shell fractures, which is consistent with previous AFM indentation experiments on hardshelled fluid-filled capsules that have shown buckling, bulging, and dimpling of the outer shell as the localized pressure increases ${ }^{[33,41-43]}$. (3) The tip completely penetrates through the shell, at which point the particles are assumed to be ruptured. (4) Following penetration, complete fracture of the shell occurs, allowing liquid to flow out. (5) Finally, as the liquid flows from the fractured particle shell, contact with ambient oxygen causes an oxide to form, fracture, and reform under the AFM tip, as indicated by the force oscillations shown in Figure 4d. We note that this single particle rupture process includes both the compressive and adhesive force as the AFM returns to its original vertical position. The adhesion forces as the AFM tip retracts are minimal compared to the bulk mechanical loading and, therefore, were not included in the following analysis ${ }^{[44]}$. 

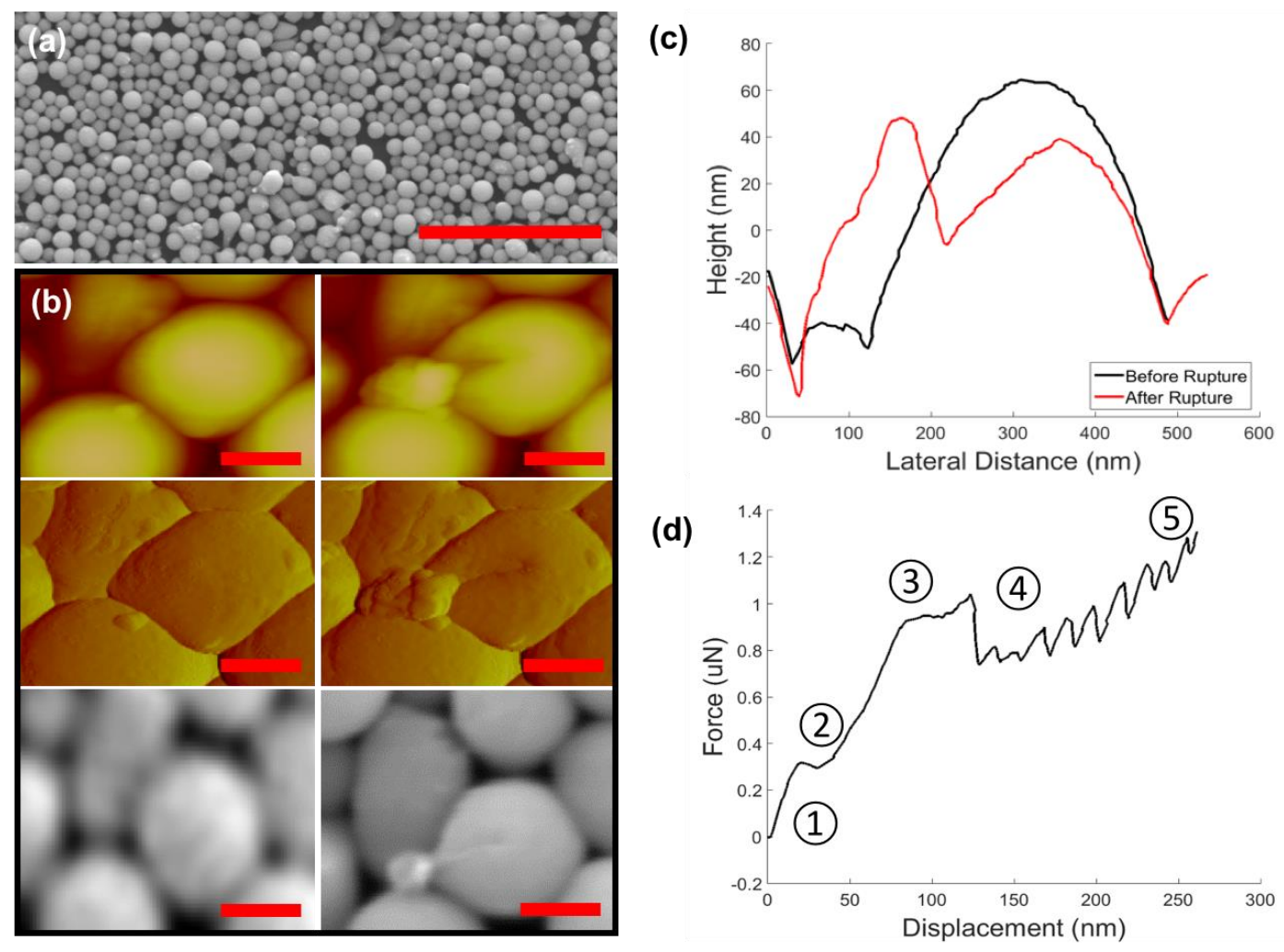

(d)

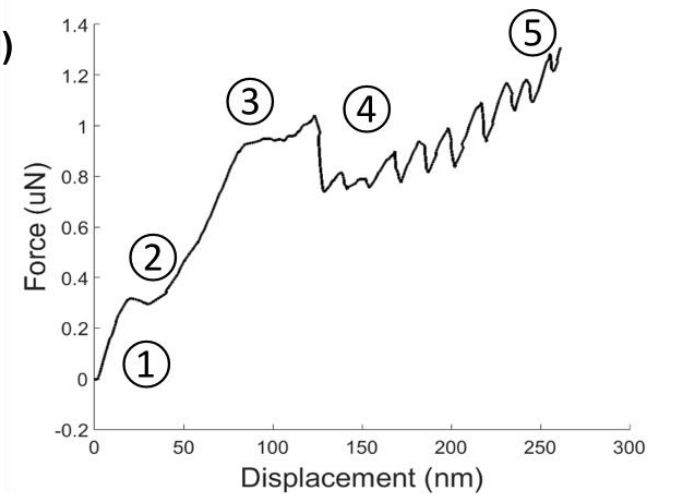

(e)

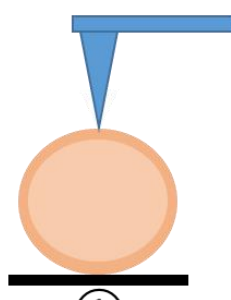

(1)

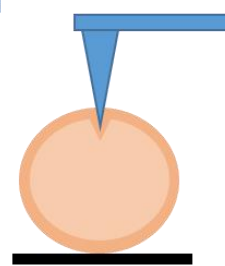

(2)

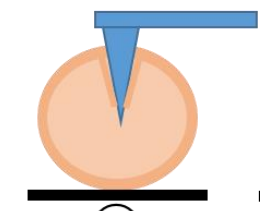

(3)

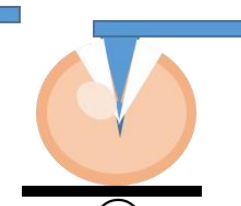

(4)

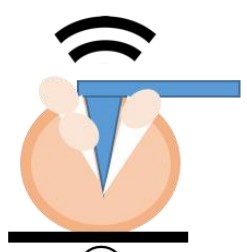

(5)

Figure 4. Schematics of the AFM experiment single particle rupture experiment. (a) Self-assembled monolayer of liquid metal nanoparticles using the Langmuir-Schaefer method. Scale bar represents $3 \mu \mathrm{m}$. (b) AFM Z map (top), AFM phase map (middle), and SEM images (bottom) of particles before (left) and after (right) rupture. Images on the right show that the particle shell is visibly broken and liquid metal has spilled out. Scale bars are $100 \mathrm{~nm}$ in length. (c) Cross-section of a single liquid metal nanoparticle before and after particle rupture. (d) Forcedisplacement curve represents a single popping test. Numbers correspond to process in (e). (e) Schematic showing particle popping sequence: 1. AFM tip makes contact with particle; 2. Viscoelastic shell of particle undergoes limited deformation; 3. AFM tip penetrates oxide shell, liquid metal is exposed; 4. Complete fracture of oxide shell, liquid starts to flow out; 5. Exposed liquid reforms shell, causing variation in force as shell resists tip motion. 
The single particle rupture data was used to verify measurements made on bulk particle films. Figure 5a shows the measured force per particle, taken as point 3 in Figure 4d-e, with respect to particle diameter. Primary sources of error come from the AFM force data that are subject to the local failure properties of the shell and imperfect centering of the tip contact at the poles of each particle ${ }^{[45]}$. Due to the differential centrifugation process used to isolate ranges of particle sizes for preparation of the monolayer, particles of less than $100 \mathrm{~nm}$ in diameter or larger than $500 \mathrm{~nm}$ were not tested. However, the AFM data further supports our claim that the trend is linear by nature and also validates the assumptions of the macroscale model, as seen by comparing the rupture force magnitude in Figures $2 \mathrm{~b}$ and $5 \mathrm{a}$ (combined in Supporting Information Figure S5). We believe that the slight difference of the slopes between the film sintering experiments and the single particle rupture experiments can be attributed to the difference in tip geometry and thus, the surface area in contact with the plate or tip ${ }^{[45-47]}$. When rupture occurs during compression between parallel plates, the contact area is much larger than with a sharp tip, where the contact area on the particle is only a small fraction of the total surface area ${ }^{[19,45]}$.

\section{Particle Shell Characterization}

The individual particle rupture experiments were further used to derive the stiffness of the particle shells. The particles can be considered as isotropically-elastic thin-shell microspheres as long as: (1) the ratio of shell thickness to radius is smaller than 0.05 , (2) the applied load is pointlike, and (3) the load is exerted at the poles of the particle. The popping experiments completed here fulfill the former two conditions and partially fulfill the third, as shown by the centered application of force on the particle in Figure $4 \mathrm{c}{ }^{[43,48]}$. Meeting these requirements, the particle shell stiffness is the slope of the force distance-curve, defined as the linear region between points (2) and (3) in Figures 4d-e:

$$
k=\frac{f}{\delta}
$$

where $\mathrm{k}$ is the stiffness of the particle shell, $f$ is the measured rupture force for a particle, and $\delta$ is the displacement of the AFM tip. A plot of resulting stiffness for all particles ruptured can be seen in Figure 5b. The average shell stiffness of all liquid metal particles tested was $16.04 \pm 4.03$ $\mathrm{N} / \mathrm{m}$, which is comparable to the stiffness of harder foams and softer polymers $(\mathrm{E} \sim 0.4 \mathrm{GPa})$. Based on these findings, we conclude that there is no correlation between the size of thiolated particles and stiffness of the thiol shell. However, the derived thiol shell stiffness is useful for both approximating the stiffness of metal oxide shells for small particle diameters $(<100 \mathrm{~nm}$, as shown in Figure 2b), as well as identifying our ability to rupture the particles on various substrates. Throughout our experiments, we have qualitatively noted that it is easier to rupture the liquid metal nanoparticles on stiffer substrates, while rupture is often not achievable on very soft substrates. We suspect that this observation is directly correlated to the stiffness of the particle shell relative to the stiffness of the substrate. For maximally flexible electronics printed on soft elastomers, larger, oxide-coated particles will minimize the effect of stiffness of the particle shell and enable rupture compatibility with soft substrates. 

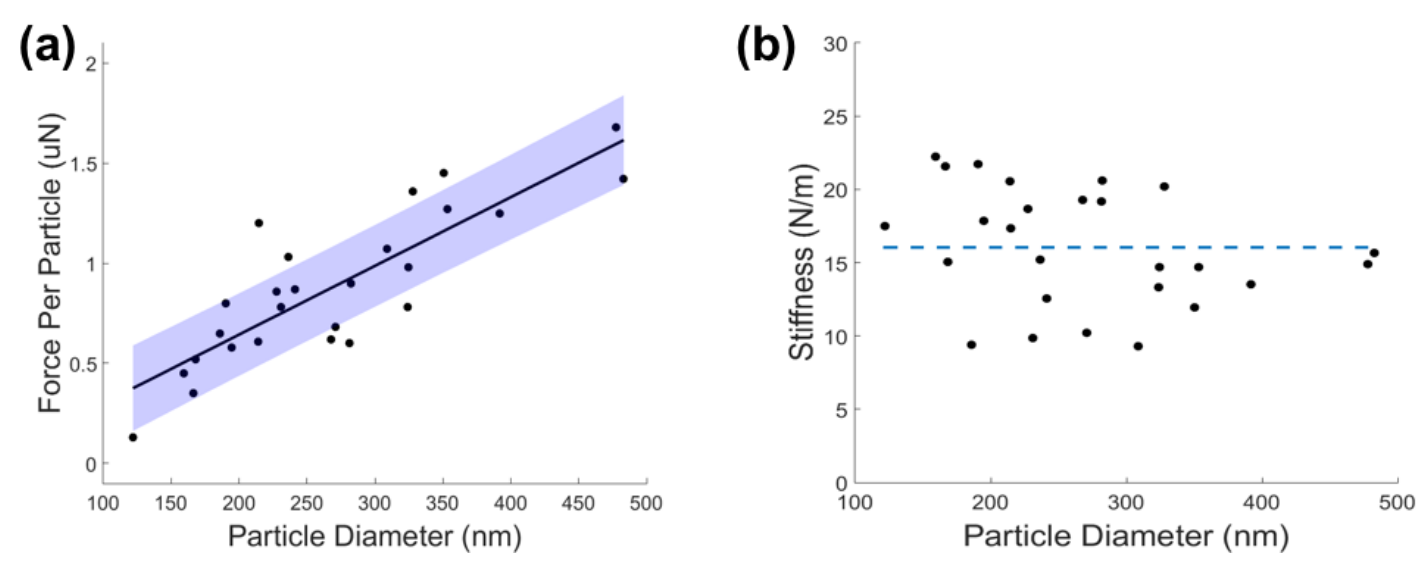

Figure 5. (a) Single particle rupture data - measured rupture force vs. particle diameter. Confidence band around data represents one standard deviation based on overall data set. Linear approximation shown. (b) Stiffness measurements for all AFM particle rupture tests. The dotted line represents the overall average.

\section{CONCLUSION}

In this study, we have extended previous characterization of liquid metal nanoparticles based on exposure to ultrasonication, specifically to achieve particles between $650 \mathrm{~nm}$ and $50 \mathrm{~nm}$ in diameter. The electrical response of thiolated and non-thiolated particle films was evaluated via mechanical compression between parallel plates, demonstrating a threshold force at which sintering may be achieved. A linear relationship between particle size and particle rupture force was derived and validated via the rupture of individual particles using AFM. Furthermore, we have derived the relationships between particle formation, core composition, shell stiffness, and conductance after rupture and coalescence. We expect these relationships to contribute to future scalable manufacturing of liquid metal-based soft electronics. 


\section{EXPERIMENTAL SECTION}

*Portions of this procedure, specifically regarding nanoparticle fabrication and film rupture testing, were originally conducted by Boley et al. ${ }^{[19]}$ They are shown here as a reference.*

\section{Liquid Metal Nanoparticle Fabrication:}

All non-thiolated liquid metal nanoparticles were made using a fixed mass $(362 \pm 5 \mathrm{mg})$ of eutectic gallium indium (EGaIn; Sigma Alrich PN 495425) deposited into a Kimble Chase 3 dram glass vial using a 3mL BD Syringe and a Nordson 14G needle (PN 7018043), weighed using a OHAUS Pioneer mass balance. Prior to the addition of materials into the glass vial, a sequential cleaning process consisting of washing and drying Liquinox cleaning solution (Alconox PN 1232), distilled water, and neat ethanol was done to prevent any interference from chemical residue on the interior of the vial. Each sample was suspended in the vial by micropipetting (BioPette Plus PN BPP1000) $4 \mathrm{~mL}$ of ethanol (Koptec PN V101). The suspended samples were made using a QSonica Q700 microtip sonicator (PN 4417) at 30\% amplitude at a fixed distance $(\approx 1 \mathrm{~mm})$ for various durations, ranging from $30 \mathrm{~min}$ to $960 \mathrm{~min}$. Parafilm was placed over the opening of the vial to prevent foreign entry. Prior to obtaining samples used for particle or film characterization, each sample was vigorously re-suspended using a Vortex-Genie 2 shaker (Cole-Parmer PN UX-04724-05) to achieve uniform particle distribution within the suspension.

For creation of thiolated liquid metal nanoparticles, a diluted thiol ethanol solution was added to the cleaned glass vials containing the fixed mass of liquid metal. The solution was created by adding a measured mass of 3-mercapto-N-nonylpropionamid thiol (Sigma Aldrich PN 686492) to a volume of ethanol and stirred vigorously until fully dissolved. Samples were sonicated for $60 \mathrm{~min}$ at room temperature before undergoing a differential centrifugation process to focus efforts on a limited range of particle sizes. The samples were first subjected to 6238 relative centrifugal force (RCF) in a micro centrifuge (Cole-Parmer PN WU-39065-05) for 20 min. The aliquot was removed and replaced by an equal volume of new ethanol, in which the pellet was resuspended using a bath sonicator (Branson M1800) and the Vortex-Genie shaker. The samples were then centrifuged again, but at only $250 \mathrm{RCF}$. This process was repeated at this RCF three times in order to achieve particle size purification. Thiolated particle shells were analyzed using $\mathrm{x}$-ray photoelectron spectroscopy (XPS), showing the presence of some oxide in the shell composition (see Table S1 in Supporting Information).

\section{Particle Characterization:}

Scanning electron microscopy (SEM) was used to determine the average particle size. After depositing $50 \mu \mathrm{L}$ of each onto cleaned (rinsed/dried with acetone, isopropanol, ethanol, water) silicon wafers (WRS Materials PN 3P0SSP-002) using a micropipette (BioPette Plus BPP200), high resolution images were obtained using an SEM (Philips XL-40 FEI, $15 \mathrm{kV}, 3 \mu \mathrm{m}$ beam spot size). The images were then processed using ImageJ (FIJI) through the following procedure: (1) Raw greyscale SEM images were converted to a binary image (Image > Adjust > Threshold, with a lower cutoff of $\approx 30$ and an upper cutoff of $\approx 230$ and dark background option); (2) each resulting image was segmented using built in ImageJ watershed function (Process > Binary > Watershed) [49]; (3) the sizes of all visible particles were calculated using ImageJ's particle analysis package (Analyze > Analyze Particles with size ranging from 10 square pixels to infinity, circularity from 0 to 1 , showing the bare outline of particles); (4) the diameter was 
computed for each particle based on a spherical approximation and then averaged for each sample.

\section{Film Rupture - Conductance Testing:}

The non-thiolated dispersion was added one drop at a time (50 $\mu \mathrm{L}$ in total) via micropipette (BioPette Plus BPP200) into a reservoir made of polydimethylsiloxane (PDMS) elastomer. After spin coating PDMS (Dow Corning Sylgard 184) with a 10:1 ratio between the base and crosslinking agent at 200 RPM using a spin coater (Specialty Coating Systems G3-8) copper wires were added to each reservoir. A second coat of PDMS was applied using the above spin coating process. Using a desktop laser system (Universal Laser Systems VLS2.30), $2.5 \mathrm{~mm}$ holes were cut using a laser ablation technique to hold the added dispersion later on (Figure S6). The samples were loaded into a single column materials tester (Instron PN 3345) fitted with a 1 $\mathrm{kN}$ load cell. The copper leads on the PDMS reservoirs were connected to a Wheatstone bridge circuit, where the other three resistors in the circuit were $1000 \Omega$ and the voltage supply at $5 \mathrm{~V}$ (Figure S7). The circuit output was connected to the materials testing unit so that the built in software package could measure the external voltage output as a function of both displacement and applied force. Samples were compressed at a steady rate of $1 \mathrm{~mm} \mathrm{~min}^{-1}$ three times for each desired test.

\section{Oxide Phase Validation:}

Non-conductive samples underwent identical compression tests using the materials tester but with a heated base. A heat sheet (McMaster-Carr PN 8009T14) was wrapped around the steel base of the materials tester and heated the sample via conduction through the material. The temperature was elevated using a programmable temperature controller (McMaster-Carr PN 4314K6) and verified with an attached thermocouple (McMaster-Carr PN 37045K192). The temperature of the materials tester base was held constant at $50{ }^{\circ} \mathrm{C}$. After depositing $50 \mu \mathrm{L}$ of dispersion into the reservoir, the compression test resumed as described above at a compression rate of $1 \mathrm{~mm} \mathrm{~min}^{-1}$.

\section{Langmuir Monolayers of Liquid Metal Nanoparticles:}

A thiolated dispersion of liquid metal nanoparticles was sonicated for one hour before centrifugation and added to a Kibron microtrough $(5 \mathrm{~cm} \times 23 \mathrm{~cm})$. As seen in Figure S4, particle size is controlled by differential centrifugation, a process that uses various g-forces to fractionate the particles. By using high forces and resuspending the precipitate, the unwanted under-sized particles can be discarded. Likewise, exposure to lower g-forces and keeping the supernatant allows for the disposal of over-size particles. This method allows us to create a monolayers of particles with minimal size variations. For each monolayer, a total of $3 \mu \mathrm{L}$ of thiolated dispersion (concentration of $50 \mathrm{mg} / \mathrm{mL}$ ) was added to the subphase one drop at a time - approximately 0.6 $\mu \mathrm{L} / \mathrm{s}$ using a micropipette (BioPette Plus BPP200). The temperature of the deionized water (18 $\mathrm{M} \Omega \mathrm{cm}$ ) in the trough was $20^{\circ} \mathrm{C}$. Once the ethanol carrier solvent evaporated, the hydrophobic thiolated liquid metal nanoparticles remained on the subphase and a reflective dark silver was observed (reflectivity points to smooth surfaces). The sample was then compressed at 10 $\mathrm{mm} / \mathrm{min}$ to improve surface coverage (Figure S8). Using the Langmuir-Schaefer method, the newly formed monolayer films were transferred onto an OTS-modified silicon wafer substrate. In order to characterize both the particle size and polydispersity of the nanoparticles and the 
films composed of them, dynamic light scattering (DLS) was employed using a Malvern Zetasizer Nano ZS at a scattering angle of $173^{\circ}$. SEM imaging was performed on the films using a NOVA nanoSEM FESEM field-emission with an accelerating voltage of 10k. The image characterization process was identical to that of the Particle Characterization section. AFM height maps were taken of each film using specifications found in Table S2.

\section{Single Particle Rupture:}

Langmuir-Schaefer monolayers were deposited onto an OTS modified silicon wafer substrate (without pt coating) as previously described. The temperature of the deionized water (18 $\mathrm{M} \Omega$ $\mathrm{cm}$ ) in the trough was $20{ }^{\circ} \mathrm{C}$. SEM observations were performed using a NOVA nanoSEM FESEM field emission (FEI) high-resolution microscope using an accelerating voltage of $10 \mathrm{kV}$. AFM topographic images and force-distance curves were acquired on a Multimode scanning probe microscope (Veeco, USA) at ambient conditions. The sharp indentation tip used was a hard-diamond-like-carbon (Figure S9). The spring constants of the probe were determined using the thermal tune method ${ }^{[50]}$. The force measurements were done using probe HQ NSC 15/Hard/AL BS (MikroMasch) cantilever with a force constant of $40 \mathrm{~N} / \mathrm{m}, \mathrm{n}$-type silicon tip $(\mathrm{h}=$ $12 \sim 18 \mu \mathrm{m}, \mathrm{r}<20 \mathrm{~nm}$ ) (Table S2). The substrate surface was reanalyzed after the tip had both come into contact and been retracted (Figure S10-S11). For the force-distance curve analysis, the sensitivity and spring constant of the cantilever were kept constant at $41.95 \pm 2.96 \mathrm{~nm} / \mathrm{V}$ and $30.40 \pm 0.44 \mathrm{~N} / \mathrm{m}$, respectively. Procedures were reviewed for: force-displacement curve measurement ${ }^{[50-52]}$, properties of nano-sized capsules ${ }^{[53]}$, adhesion force curve analysis ${ }^{[54]}$, Atomic J software [55], AFM mechanical properties [56], AFM colloid properties [57], and measurements of rigidity ${ }^{[58]}$.

\section{ACKNOWLEDGMENTS}

We would like to thank Michelle Yuen for her willingness to help with MATLAB coding and experiment design, Adam Bilodeau for his help with SEM, and Mohammed Mohammed for his suggestions regarding various particle characterization methods and figure preparation.

This work was partially supported by a National Science Foundation CAREER Award (1454284). T.L was partially funded by the William K. \& Gail E. Cordier Fellowship from the Purdue University School of Mechanical Engineering.

\section{AUTHOR CONTRIBUTIONS}

T.L conducted the film experiments and discussed results with E.W.. S.H. and J.W.B. conducted experiments with the Langmuir trough and AFM. T.L wrote the manuscript with help from S.H. and R.K.. Further discussions and theories were developed with D.T. and R.K.. 


\section{REFERENCES}

[1] Park, Y.-L., Chen, B.-R., Wood, R.J., 2012. Design and fabrication of soft artificial skin using embedded microchannels and liquid conductors. Sensors Journal, IEEE 12, 2711-2718.

[2] Park, Y.-L., Majidi, C., Kramer, R., Bérard, P., Wood, R.J., 2010. Hyperelastic pressure sensing with a liquid-embedded elastomer. Journal of Micromechanics and Microengineering 20, 125029. doi:10.1088/0960-1317/20/12/125029

[3] Vogt, D.M., Park, Y.-L., Wood, R.J., 2013. Design and characterization of a soft multi-axis force sensor using embedded microfluidic channels. Sensors Journal, IEEE 13, 4056-4064.

[4] Finkenauer, L.R., Majidi, C., 2014. Complaint liquid metal electrodes for dielectric elastomer actuators. p. 90563I-90563I-7. doi:10.1117/12.2049112

[5] Reus, W.F., Thuo, M.M., Shapiro, N.D., Nijhuis, C.A., Whitesides, G.M., 2012. The SAM, Not the Electrodes, Dominates Charge Transport in Metal-Monolayer//Ga2O3/Gallium-Indium Eutectic Junctions. ACS Nano 6, 4806-4822. doi:10.1021/nn205089u

[6] Blaiszik, B.J., Jones, A.R., Sottos, N.R., White, S.R., 2014. Microencapsulation of galliumindium (Ga-In) liquid metal for self-healing applications. Journal of Microencapsulation 31, 350-354. doi:10.3109/02652048.2013.858790

[7] Zhang, J., Yao, Y., Liu, J., 2015. Autonomous convergence and divergence of the selfpowered soft liquid metal vehicles. Sci. Bull. 60, 943-951. doi:10.1007/s11434-015-0786-Z

[8] Kramer, R.K., Majidi, C., Sahai, R., Wood, R.J., 2011. Soft curvature sensors for joint angle proprioception, in: 2011 IEEE/RSJ International Conference on Intelligent Robots and Systems. IEEE, pp. 1919-1926.

[9] Kramer, R.K., Majidi, C., Wood, R.J., 2011. Wearable tactile keypad with stretchable artificial skin, in: Robotics and Automation (ICRA), 2011 IEEE International Conference on. IEEE, pp. 1103-1107.

[10] Ponce Wong, R.D., Posner, J.D., Santos, V.J., 2012. Flexible microfluidic normal force sensor skin for tactile feedback. Sensors and Actuators A: Physical 179, 62-69.

doi:10.1016/j.sna.2012.03.023

[11] Gozen, B.A., Tabatabai, A., Ozdonganlar, O.B., Majidi, C., 2014. High-density soft-matter electronics with micron-scale line width. Advanced Materials 26, 5211-5216.

doi:10.1002/adma.201400502

[12] Kramer, R.K., Majidi, C., Wood, R.J., 2013. Masked Deposition of Gallium-Indium Alloys for Liquid-Embedded Elastomer Conductors. Advanced Functional Materials 23, 5292-5296. doi:10.1002/adfm.201203589 
[13] Boley, J.W., White, E.L., Chiu, G.T.-C., Kramer, R.K., 2014. Direct Writing of GalliumIndium Alloy for Stretchable Electronics. Advanced Functional Materials 24, 3501-3507. doi:10.1002/adfm.201303220

[14] Ladd, C., So, J.-H., Muth, J., Dickey, M.D., 2013. 3D Printing of Free Standing Liquid Metal Microstructures. Advanced Materials 25, 5081-5085. doi:10.1002/adma.201301400

[15] Zhang, Q., Zheng, Y., Liu, J., 2012. Direct Writing of Electronics Based on Alloy and Metal (DREAM) Ink: A Newly Emerging Area and Its Impact on Energy, Environment, and Health Sciences. Frontiers in Energy 6, 311-340. doi:10.1007/s11708-012-0214-X

[16] Chiechi, R.C., Weiss, E.A., Dickey, M.D., Whitesides, G.M., 2008. Eutectic GalliumIndium (EGaIn): A Moldable Liquid Metal for Electrical Characterization of Self-Assembled Monolayers. Angewandte Chemie International Edition 47, 142-144. doi:10.1002/anie.200703642

[17] Hohman, J.N., Kim, M., Wadsworth, G.A., Bednar, H.R., Jiang, J., LeThai, M.A., Weiss, P.S., 2011. Directing Substrate Morphology via Self-Assembly: Ligand-Mediated Scission of Gallium-Indium Microspheres to the Nanoscale. Nano Lett. 11, 5104-5110. doi:10.1021/nl202728j

[18] Kim, E., Baek, J., 2012. Numerical study on the effects of non-dimensional parameters on drop-on-demand droplet formation dynamics and printability range in the up-scaled model.

Physics of Fluids 24, 082103. doi:10.1063/1.4742913

[19] Boley, J.W., White, E.L., Kramer, R.K., 2015. Mechanically Sintered Gallium-Indium Nanoparticles. Adv. Mater. 27, 2355-2360. doi:10.1002/adma.201404790

[20] Regan, M.J., Tostmann, H., Pershan, P.S., Magnussen, O.M., DiMasi, E., Ocko, B.M., Deutsch, M., 1997. X-ray study of the oxidation of liquid-gallium surfaces. Physical Review B $55,10786$.

[21] Dickey, M.D., Chiechi, R.C., Larsen, R.J., Weiss, E.A., Weitz, D.A., Whitesides, G.M., 2008. Eutectic Gallium-Indium (EGaIn): A Liquid Metal Alloy for the Formation of Stable Structures in Microchannels at Room Temperature. Advanced Functional Materials 18, 10971104. doi:10.1002/adfm.200701216

[22] Raabe, D., Hessling, D., 2010. Synthesis of hollow metallic particles via ultrasonic treatment of a metal emulsion. Scripta Materialia 62, 690-692. doi:10.1016/j.scriptamat.2010.01.028

[23] Larsen, R.J., Dickey, M.D., Whitesides, G.M., Weitz, D.A., 2009. Viscoelastic properties of oxide-coated liquid metals. Journal of Rheology (1978-present) 53, 1305-1326. doi:10.1122/1.3236517 
[24] Weiss, E.A., Porter, V.J., Chiechi, R.C., Geyer, S.M., Bell, D.C., Bawendi, M.G., Whitesides, G.M., 2008. The Use of Size-Selective Excitation To Study Photocurrent through Junctions Containing Single-Size and Multi-Size Arrays of Colloidal CdSe Quantum Dots. J. Am. Chem. Soc. 130, 83-92. doi:10.1021/ja076439+

[25] Zhang, W., Ou, J.Z., Tang, S.-Y., Sivan, V., Yao, D.D., Latham, K., Khoshmanesh, K., Mitchell, A., O’Mullane, A.P., Kalantar-zadeh, K., 2014. Liquid Metal/Metal Oxide Frameworks. Adv. Funct. Mater. 24, 3799-3807. doi:10.1002/adfm.201304064

[26] Suslick, K.S., 1990. Acoustic Cavitation in Homogeneous Liquids. Science. 247 (4949), 1439-1447. Doi:10.1126/science.247.4949.1439

[27] Sutherland, D.N., 1977. Random packing of circles in a plane. Journal of Colloid and Interface Science 60, 96-102.

[28] Zhang, Z., 1999. Mechanical strength of single microcapsules determined by a novel micromanipulation technique. Journal of Microencapsulation 16, 117-124. doi:10.1080/026520499289365

[29] Mercadé-Prieto, R., Allen, R., Zhang, Z., York, D., Preece, J.A., Goodwin, T.E., 2012. Failure of elastic-plastic core-shell microcapsules under compression. AIChE J. 58, 2674-2681. doi:10.1002/aic.12804

[30] Yarema, M., Wörle, M., Rossell, M.D., Erni, R., Caputo, R., Protesescu, L., Kravchyk, K.V., Dirin, D.N., Lienau, K., von Rohr, F., Schilling, A., Nachtegaal, M., Kovalenko, M.V., 2014. Monodisperse Colloidal Gallium Nanoparticles: Synthesis, Low Temperature Crystallization, Surface Plasmon Resonance and Li-Ion Storage. Journal of the American Chemical Society 136, 12422-12430. doi:10.1021/ja506712d

[31] Schön, G., 1973. Auger and direct electron spectra in X-ray photoelectron studies of zinc, zinc oxide, gallium and gallium oxide. Journal of Electron Spectroscopy and Related Phenomena 2, 75-86. doi:10.1016/0368-2048(73)80049-0

[32] Lin, Y., Cooper, C., Wang, M., Adams, J.J., Genzer, J., Dickey, M.D., 2015. Handwritten, Soft Circuit Boards and Antennas Using Liquid Metal Nanoparticles. Small 11, 6397-6403. doi:10.1002/smll.201502692

[33] Ren, L., Zhuang, J., Casillas, G., Feng, H., Liu, Y., Xu, X., Liu, Y., Chen, J., Du, Y., Jiang, L., Dou, S.X., 2016. Nanodroplets for Stretchable Superconducting Circuits. Advanced Functional Materials. doi:10.1002/adfm.201603427

[34] Matsuyama, T., Ohtsuka, M., Yamamoto, H., 2008. Measurement of Force Curve due to Electrostatic Charge on a Single Particle using Atomic Force Microscope [Translated] ${ }^{\dagger}$. KONA Powder and Particle Journal 26, 238-245. doi:10.14356/kona.2008021 
[35] Baclayon, M., Shoemaker, G.K., Uetrecht, C., Crawford, S.E., Estes, M.K., Prasad, B.V.V., Heck, A.J.R., Wuite, G.J.L., Roos, W.H., 2011. Prestress Strengthens the Shell of Norwalk Virus Nanoparticles. Nano Letters 11, 4865-4869. doi:10.1021/nl202699r

[36] Guo, D., Li, J., Xie, G., Wang, Y., Luo, J., 2014. Elastic Properties of Polystyrene Nanospheres Evaluated with Atomic Force Microscopy: Size Effect and Error Analysis. Langmuir 30, 7206-7212. doi:10.1021/la501485e

[37] Huang, S., Minami, K., Sakaue, H., Shingubara, S., Takahagi, T., 2004. Effects of the Surface Pressure on the Formation of Langmuir-Blodgett Monolayer of Nanoparticles. Langmuir 20, 2274-2276. doi:10.1021/1a0302293

[38] Sarrazin, B., Tsapis, N., Mousnier, L., Taulier, N., Urbach, W., Guenoun, P., 2016. AFM Investigation of Liquid-Filled Polymer Microcapsules Elasticity. Langmuir 32, 4610-4618. doi:10.1021/acs.langmuir.6b00431

[39] Arfsten, J., Bradtmöller, C., Kampen, I., Kwade, A., 2008. Compressive testing of single yeast cells in liquid environment using a nanoindentation system. Journal of Materials Research 23, 3153-3160. doi:10.1557/JMR.2008.0383

[40] Rong, W., Pelling, A.E., Ryan, A., Gimzewski, J.K., Friedlander, S.K., 2004.

Complementary TEM and AFM Force Spectroscopy to Characterize the Nanomechanical Properties of Nanoparticle Chain Aggregates. Nano Letters 4, 2287-2292.

doi:10.1021/n10487368

[41] Dubreuil, F., Elsner, N., Fery, A., 2003. Elastic properties of polyelectrolyte capsules studied by atomic-force microscopy and RICM. The European Physical Journal E - Soft Matter 12, 215-221. doi:10.1140/epje/i2003-10056-0

[42] Vinogradova, O.I., 2004. Mechanical properties of polyelectrolyte multilayer microcapsules. Journal of Physics: Condensed Matter 16, R1105-R1134. doi:10.1088/09538984/16/32/R01

[43] Glynos, E., Sboros, V., Koutsos, V., 2009. Polymeric thin shells: Measurement of elastic properties at the nanometer scale using atomic force microscopy. Materials Science and Engineering: B 165, 231-234. doi:10.1016/j.mseb.2009.06.016

[44] Briscoe, B.J., Liu, K.K., Williams, D.R., 1998. Adhesive Contact Deformation of a Single Microelastomeric Sphere. Journal of Colloid and Interface Science 200, 256-264. doi:10.1006/jcis.1997.5365

[45] Mercadé-Prieto, R., Zhang, Z., 2012. Mechanical characterization of microspheres capsules, cells and beads: a review. Journal of Microencapsulation 29, 277-285. doi:10.3109/02652048.2011.646331 
[46] Ahmad*, M.R., Nakajima, M., Kojima, S., Homma, M., Fukuda, T., 2008. The Effects of Cell Sizes, Environmental Conditions, and Growth Phases on the Strength of Individual W303 Yeast Cells Inside ESEM. IEEE Transactions on NanoBioscience 7, 185-193. doi:10.1109/TNB.2008.2002281

[47] Mercadé-Prieto, R., Nguyen, B., Allen, R., York, D., Preece, J.A., Goodwin, T.E., Zhang, Z., 2011. Determination of the elastic properties of single microcapsules using micromanipulation and finite element modeling. Chemical Engineering Science 66, 2042-2049. doi:10.1016/j.ces.2011.01.015

[48] Reissner, E., 1946. Stresses and Small Displacements of Shallow Spherical Shells. I. Journal of Mathematics and Physics 25, 80-85. doi:10.1002/sapm194625180

[49] Vincent, L., Soille, P., 1991. Watersheds in digital spaces: an efficient algorithm based on immersion simulations. IEEE Transactions on Pattern Analysis and Machine Intelligence 13, 583-598. doi:10.1109/34.87344

[50] Cappella, B., Dietler, G., 1999. Force-distance curves by atomic force microscopy. Surface Science Reports 34, 1-104. doi:10.1016/S0167-5729(99)00003-5

[51] Hutter, J.L., Bechhoefer, J., 1993. Calibration of atomic-force microscope tips. Review of Scientific Instruments 64, 1868. doi:10.1063/1.1143970

[52] Roos, W.H., Bruinsma, R., Wuite, G.J.L., 2010. Physical virology. Nature Physics 6, 733743. doi:10.1038/nphys 1797

[53] Dunér, G., Thormann, E., Dėdinaite, A., Claesson, P.M., Matyjaszewski, K., Tilton, R.D., 2012. Nanomechanical mapping of a high curvature polymer brush grafted from a rigid nanoparticle. Soft Matter 8, 8312. doi:10.1039/c2sm26086g

[54] Lee, H., Veerapandian, M., Kim, B.T., Yun, K., Seo, S.-W., 2012. Functional nanoparticles translocation into cell and adhesion force curve analysis. J Nanosci Nanotechnol 12, 7752-7763.

[55] Hermanowicz, P., Sarna, M., Burda, K., Gabryś, H., 2014. AtomicJ: an open source software for analysis of force curves. Rev Sci Instrum 85, 063703. doi:10.1063/1.4881683

[56] Choi, S., Cheong, Y., Lee, H.-J., Lee, S.J., Jin, K.-H., Park, H.-K., 2011. AFM study for morphological and mechanical properties of human scleral surface. J Nanosci Nanotechnol 11, 6382-6388.

[57] Li, S., Palmer, A.F., 2005. Structure and Mechanical Response of Self-Assembled Poly(butadiene)- $b$-poly(ethylene oxide) Colloids Probed by Atomic Force Microscopy. Macromolecules 38, 5686-5698. doi:10.1021/ma047858j 
[58] Dokukin, M.E., Sokolov, I., 2012. On the Measurements of Rigidity Modulus of Soft Materials in Nanoindentation Experiments at Small Depth. Macromolecules 45, 4277-4288. doi:10.1021/ma202600b 